\title{
The sum of the Möbius function
}

\author{
H. Maier and H. L. Montgomery
}

\section{ABSTRACT}

We derive from the Riemann Hypothesis an estimate for $M(x)=\sum_{n \leqslant x} \mu(n)$. This is the first improvement of the bound that Titchmarsh established in 1927.

\section{Introduction}

Let $M(x)=\sum_{1 \leqslant n \leqslant x} \mu(n)$, where $\mu(n)$ denotes the Möbius function. In [3], Littlewood proved that if the Riemann hypothesis $(\mathrm{RH})$ is true then $1 / \zeta(1 / 2+\varepsilon+i t) \ll t^{\varepsilon}$ for any fixed $\varepsilon>0$, and it follows by Perron's formula that

$$
M(x) \ll x^{1 / 2+\varepsilon} .
$$

The converse is trivial, since this estimate, by partial summation, implies that the series $\sum_{n=1}^{\infty} \mu(n) n^{-s}=1 / \zeta(s)$ converges for $\sigma>1 / 2$. Subsequently, Landau [2] showed, still assuming $\mathrm{RH}$, that (1) is valid with $\varepsilon \ll(\log \log \log x) / \log \log x$, and Titchmarsh [15] improved this to $\varepsilon \ll 1 / \log \log x$. Titchmarsh's analysis was based on the estimate

$$
\zeta\left(\frac{1}{2}+\frac{1}{\log \log t+i t}\right)^{-1} \ll \exp \left(\frac{c \log t}{\log \log t}\right) \quad(t \geqslant 4)
$$

that Littlewood [4] derived from RH. The above still stands as the best known estimate of its kind but, from the work of Selberg [13], we know that a much better estimate applies for most $t$. In particular, Selberg (unpublished) derived asymptotic formulae for the moments

$$
\int_{0}^{T}(\log |\zeta(\sigma+i t)|)^{2 k} d t
$$

from which it can be seen that the distribution of

$$
\frac{\log |\zeta(1 / 2+i t)|}{\sqrt{\log \log t}}
$$

for $4 \leqslant t \leqslant T$, tends weakly to normal distribution with mean 0 and variance $1 / 2$ as $T \rightarrow \infty$. By utilizing Selberg's techniques, we can bound the frequency with which $|\log \zeta(\sigma+i t)|$ is large. Once our basic result is in place, it can be put to various uses. For example, in Corollary 1 below, we find that $(1)$ holds with $\varepsilon=(\log x)^{-22 / 61}$.

Theorem (Assume RH). For any $x \geqslant 2$ there is a piecewise linear contour lying in the rectangle $1 / 2<\sigma<1$ and $-x \leqslant t \leqslant x$ that links the bottom edge of the rectangle to the top, for which the following estimates apply:

$$
\int_{0 \leqslant t \leqslant 16}\left|\frac{x^{s}}{\zeta(s)}\right||d s| \ll x^{1 / 2} \log \log x .
$$

Received 10 September 2007; revised 31 January 2008; published online 6 March 2009.

2000 Mathematics Subject Classification 11N56, 11M26.

Research supported in part by NSF FRG Grant DMS-0244660 and NSF Grant DMS-0653529. 
For $16 \leqslant T \leqslant \exp \left((\log x)^{39 / 61}\right)$, we have

$$
\int_{T \leqslant t \leqslant 2 T}\left|\frac{x^{s}}{\zeta(s)}\right||d s| \ll x^{1 / 2} T\left(\frac{e \log x}{\log T}\right)^{C \log T / \log \log T} .
$$

For $\exp \left((\log x)^{39 / 61}\right) \leqslant T \leqslant x$, we have

$$
\int_{T \leqslant t \leqslant 2 T}\left|\frac{x^{s}}{\zeta(s)}\right||d s| \ll x^{1 / 2} T(\log x)^{A} \exp \left(\left(\frac{\log x}{\log T}\right)^{39 / 22}\right) .
$$

Finally, for each real number $t$, where $\exp \left((\log x)^{39 / 61}\right) \leqslant t \leqslant x$, let $\sigma(t)$ be chosen so that $\sigma(t)+i t \in$. Then the quantity

$$
\frac{x^{\sigma}}{|\zeta(\sigma+i t)|}
$$

is an increasing function of $\sigma$ for $\sigma(t) \leqslant \sigma<\infty$.

In the above, and elsewhere, we denote by $A$ and $C$ effectively computable absolute constants, which may be different from one occasion to the next. The limit of our method would allow the exponent $39 / 22$ to be replaced by a slightly smaller (presumably transcendental) number, but, for simplicity, we content ourselves with the above.

Corollary 1 (Assume RH). For $x \geqslant 2$, we have

$$
M(x) \ll x^{1 / 2} \exp \left(C(\log x)^{39 / 61}\right) .
$$

Corollary 2 (Assume RH). There is an absolute constant $A$ such that, if $x \geqslant 2$ and $h \leqslant x^{1-\delta}$, then

$$
\sum_{x<n \leqslant x+h} \mu(n) \ll_{\delta} x^{1 / 2}(\log x)^{A}
$$

The above estimates are remarkably inferior to the corresponding estimates for $\pi(x)$ : assuming RH, we know that $\pi(x)=\operatorname{li} x+\mathrm{O}\left(x^{1 / 2} \log x\right)$ (see [9, Theorem 13.1]). In the opposite direction it is easy to prove that $M(x)=\Omega_{ \pm}\left(x^{1 / 2}\right)$. Mertens conjectured that $|M(x)| \leqslant x^{1 / 2}$, but this has been disproved by Odlyzko and te Riele [11] by means of extensive numerical calculations. The finer behavior of $M(x)$ depends in a complicated way both on the distribution of $\left|\zeta^{\prime}(\rho)\right|$ as $\rho=1 / 2+i \gamma$ runs over the nontrivial zeros of the zeta function, and on the extent of linear independence of the imaginary parts of the $\gamma>0$. Based on speculations relating to random matrix theory, Hughes, Keating, and O'Connell $[\mathbf{1}]$ have conjectured that

$$
\sum_{0<\gamma \leqslant T} \frac{1}{\left|\zeta^{\prime}(\rho)\right|} \sim \alpha T(\log T)^{1 / 4},
$$

where $\alpha$ is a certain specified positive constant. This estimate implies (assuming $\mathrm{RH}$ ) that

$$
M(x) \ll x^{1 / 2}(\log x)^{5 / 4}
$$

for $x \geqslant 2$. However, more should be true, and indeed Gonek (unpublished) conjectured that

$$
\varlimsup_{x \rightarrow \infty} \frac{M(x)}{x^{1 / 2}(\log \log \log x)^{5 / 4}} \gtrless 0
$$


(see [10]). This corresponds to the conjecture of Montgomery [7] that

$$
\varlimsup_{x \rightarrow \infty} \frac{\psi(x)-x}{x^{1 / 2}(\log \log \log x)^{2}}= \pm \frac{1}{2 \pi} .
$$

\section{Monotonicity principles}

The following lemmas capture in a succinct manner some of the main ingredients in the arguments of Landau [2] and Titchmarsh [15].

Lemma 1 (Assume RH). Let $\xi(s)=(1 / 2) s(s-1) \zeta(s) \Gamma(s / 2) \pi^{-s / 2}$. Then, for any fixed $t$,

$$
\left(\sigma-\frac{1}{2}\right) \Re \frac{\xi^{\prime}}{\xi}(\sigma+i t)
$$

is a non-negative strictly increasing function of $\sigma$ for $1 / 2 \leqslant \sigma<\infty$.

By the symmetry of the functional equation $\xi(1-s)=\xi(s)$, it follows that expression (9) is invariant when $\sigma$ is replaced by $1-\sigma$. Thus, for $\sigma \leqslant 1 / 2$, the expression is non-negative and strictly decreasing.

Proof. By Montgomery and Vaughan [9, (10.28) and (10.30)], we know that

$$
\Re \frac{\xi^{\prime}}{\xi}(s)=\sum_{\rho} \Re \frac{1}{s-\rho},
$$

where the sum is over all nontrivial zeros $\rho=1 / 2+i \gamma$ of the zeta function. Hence expression (9) is equal to

$$
\sum_{\gamma} \frac{(\sigma-1 / 2)^{2}}{(\sigma-1 / 2)^{2}+(t-\gamma)^{2}}
$$

Since each term of this sum has the required properties, the result is immediate.

Lemma 2. There is a constant $C>0$ such that, if $t \geqslant C$, then

$$
\left(\sigma-\frac{1}{2}\right)\left(\Re \frac{\zeta^{\prime}}{\zeta}(\sigma+i t)-\Re \frac{\xi^{\prime}}{\xi}(\sigma+i t)+\frac{1}{2} \log \frac{t}{2}\right)
$$

is a non-negative strictly increasing function of $\sigma$ for $1 / 2 \leqslant \sigma \leqslant 2$.

It seems likely that $C=2.8$ is admissible in the above. Certainly $C=2.7$ is not.

Proof. Since

$$
\frac{\zeta^{\prime}}{\zeta}(s)=\frac{\xi^{\prime}}{\xi}(s)-\frac{1}{s-1}+\frac{1}{2} \log \pi-\frac{1}{2} \frac{\Gamma^{\prime}}{\Gamma}\left(\frac{s}{2}+1\right),
$$

we need to show that

$$
\frac{1}{2}\left(\sigma-\frac{1}{2}\right)\left(\frac{2(1-\sigma)}{(\sigma-1)^{2}+t^{2}}+\log \frac{\pi t}{2}-\Re \frac{\Gamma^{\prime}}{\Gamma}\left(\frac{\sigma}{2}+1+\frac{i t}{2}\right)\right)
$$

is non-negative and increasing. By the Euler-MacLaurin sum formula, we find that

$$
\frac{\Gamma^{\prime}}{\Gamma}(s)=\log s+\mathrm{O}(1 /|s|)
$$


uniformly for $|\arg s| \leqslant \pi-\delta$ and $|s| \geqslant 1$ (see [9, Theorem C.1]). By applying Cauchy's formula to $\left(\Gamma^{\prime} / \Gamma\right)(s)-\log s$, or by applying the Euler-MacLaurin formula to the expansion

$$
\left(\frac{\Gamma^{\prime}}{\Gamma}\right)^{\prime}(s)=\sum_{n=0}^{\infty} \frac{1}{(s+n)^{2}}
$$

we also find that

$$
\left(\frac{\Gamma^{\prime}}{\Gamma}\right)^{\prime}(s)=\frac{1}{s}+\mathrm{O}\left(\frac{1}{|s|}^{2}\right)
$$

uniformly for $|\arg s| \leqslant \pi-\delta$ and $|s| \geqslant 1$. By appealing to these estimates, we discover that the derivative of (11) with respect to $\sigma$ is $(1 / 2) \log \pi+\mathrm{O}(1 / t)$, which is positive if $t \geqslant C$, and so expression (11) is strictly increasing. Since it vanishes when $\sigma=1 / 2$, it also follows that it is non-negative.

On summing the quantities in the two preceding lemmas, we obtain the following.

Lemma 3 (Assume RH). There is an absolute constant $C>0$ such that, if $t \geqslant C$, then

$$
\left(\sigma-\frac{1}{2}\right)\left(\Re \frac{\zeta^{\prime}}{\zeta}(\sigma+i t)+\frac{1}{2} \log \frac{t}{2}\right)
$$

is a non-negative strictly increasing function of $\sigma$ for $1 / 2 \leqslant \sigma \leqslant 2$.

Lemma 4 (Assume RH). If $1 / 2<\sigma_{1} \leqslant \sigma_{2} \leqslant 2$ and $t \geqslant C$, then

$$
\left|\zeta\left(\sigma_{1}+i t\right)\right| \geqslant\left|\zeta\left(\sigma_{2}+i t\right)\right|\left(\frac{\sigma_{1}-1 / 2}{\sigma_{2}-1 / 2}\right)^{\left(\sigma_{2}-1 / 2\right)\left(\Re\left(\zeta^{\prime} / \zeta\right)\left(\sigma_{2}+i t\right)+(1 / 2) \log (t / 2)\right)} .
$$

Proof. Let $f(\sigma)$ denote expression (12). Thus $f(\sigma) \leqslant f\left(\sigma_{2}\right)$ for $1 / 2 \leqslant \sigma \leqslant \sigma_{2}$. We divide both sides of this by $\sigma-1 / 2$ and integrate over $\sigma_{1} \leqslant \sigma \leqslant \sigma_{2}$, exponentiate, and discard a factor $(t / 2)^{\left(\sigma_{1}-\sigma_{2}\right) / 2}$ to obtain the stated inequality.

Littlewood [4] showed (assuming RH) that

$$
\log \zeta(s) \ll \frac{\log \tau}{\log \log \tau}, \quad \Re \frac{\zeta^{\prime}}{\zeta}(s) \ll \log \tau
$$

for $\sigma \geqslant 1 / 2+1 / \log \log \tau$, where $\tau=|t|+4$. Suppose that $4 \leqslant t \leqslant x$, and take

$$
\sigma_{1}=\frac{1}{2}+\frac{\log t}{(\log x) \log \log t}, \quad \sigma_{2}=\frac{1}{2}+\frac{1}{\log \log t}
$$

in Lemma 4. Then, by the estimates (13), we see that

$$
\frac{1}{\zeta(s)} \ll\left(\frac{e \log x}{\log t}\right)^{\frac{C \log t}{\log \log t}}
$$

for $s=\sigma_{1}+i t$. Titchmarsh's estimate follows immediately by applying Perron's formula on this contour.

\section{Large value estimates}

We first establish a basic tool. 
Lemma 5. Suppose that

$$
S(s)=\sum_{p \leqslant N} a(p) p^{-s},
$$

where the $a(p)$ are arbitrary real or complex numbers. Suppose that $\alpha, T$, and $T_{0}$ are real numbers such that $T \geqslant 2$. For $1 \leqslant r \leqslant R$, let $s_{r}=\sigma_{r}+i t_{r}$ be points such that $\sigma_{r} \geqslant \alpha$ and $T_{0} \leqslant t_{r} \leqslant T_{0}+T$. Also, suppose that these points are well spaced to the extent that $\mid s_{r_{1}}-$ $s_{r_{2}} \mid \geqslant 1 / \log T$ for $1 \leqslant r_{1}<r_{2} \leqslant R$. If $k$ is a positive integer such that $N^{k} \leqslant T$, then

$$
\sum_{r=1}^{R}\left|S\left(s_{r}\right)\right|^{2 k} \ll T(\log T)^{2} k !\left(\sum_{p \leqslant N}|a(p)|^{2} p^{-2 \alpha}\right)^{k} .
$$

Proof. Let $D(s)=S(s)^{k}=\sum_{n \leqslant N^{k}} c_{n} n^{-s}$. We show first that

$$
\sum_{r=1}^{R}\left|D\left(s_{r}\right)\right|^{2} \ll T(\log T)^{2} \sum_{n}\left|c_{n}\right|^{2} n^{-2 \alpha} .
$$

To this end, let $(a)$ denote a disc of radius $1 /(2 \log T)$ centered at $a$. Then

$$
|D(s)|^{2} \leqslant \frac{4}{\pi}(\log T)^{2} \iint_{(s)}|D(x+i y)|^{2} d x d y
$$

for any $s$. Since the discs $\left(s_{r}\right)$ are disjoint and since they all lie in the half-strip $\sigma \geqslant \alpha-1 /$ $\log T$ and $T_{0}-1 \leqslant t \leqslant T_{0}+T+1$, it follows that

$$
\sum_{r=1}^{R}\left|D\left(s_{r}\right)\right|^{2} \ll(\log T)^{2} \int_{\alpha-1 / \log T}^{\infty} \int_{T_{0}-1}^{T_{0}+T+1}|D(\sigma+i t)|^{2} d t d \sigma .
$$

By a standard mean-value theorem (see $[\mathbf{5}$, Theorem $6.1 ; \mathbf{6}, \mathbf{8}]$ ), we know that the inner integral above is

$$
\left(T+\mathrm{O}\left(N^{k}\right)\right) \sum_{n}\left|c_{n}\right|^{2} n^{-2 \sigma} .
$$

On integrating this with respect to $\sigma$, we find that

$$
\sum_{r=1}^{R}\left|D\left(s_{r}\right)\right|^{2} \ll T(\log T)^{2} \sum_{n} \frac{\left|c_{n}\right|^{2}}{n^{2 \alpha} \log n} .
$$

Note that the term $n=1$ does not occur in the above, since $c_{n} \neq 0$ only when $\Omega(n)=k$. Thus $\log n \gg 1$ for all the above $n$, and hence we have (14).

To complete the proof, we note that, if $n$ has the canonical factorization $n=p_{1}^{k_{1}} p_{2}^{k_{2}} \cdot \ldots \cdot p_{m}^{k_{m}}$ with $\Omega(n)=\sum_{i} k_{i}=k$, then

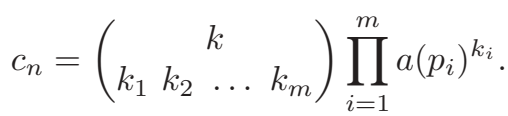

Hence

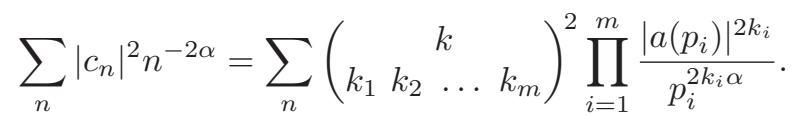

Here the multinomial coefficient is at most $k$ ! in all cases, and so the above is at most

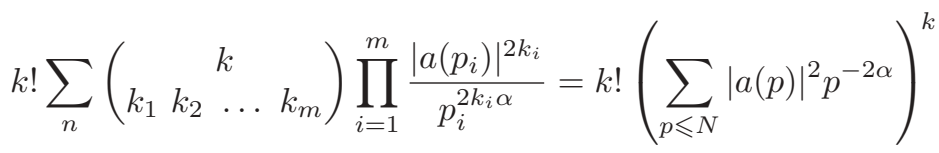


by the multinomial theorem. The stated result now follows by combining the above with (14).

We now use the above, and Selberg's technique, to estimate the number of times that $\left|\left(\zeta^{\prime} / \zeta\right)(s)\right|$ is large. It transpires that the exponent $\xi$ in our Theorem 1 depends on the constants that arise in the next lemma, and so we take care to optimize the parameters. The main parameter, $\eta$, is left undetermined until its optimal value becomes apparent, in the next section.

Lemma 6 (Assume RH). Suppose that $0<\eta \leqslant 1 / 2$, that $\varepsilon>0$, that $T \geqslant T_{0}(\varepsilon)$, and that $\alpha \geqslant 1 / 2+1 / \log T$. For $1 \leqslant r \leqslant R$, let $s_{r}=\sigma_{r}+i t_{r}$ be points such that $\sigma_{r} \geqslant \alpha$ and $T \leqslant t_{r} \leqslant$ $2 T$, with the $t_{r}$ well spaced in the sense that $\left|t_{r_{1}}-t_{r_{2}}\right| \geqslant 1$ whenever $r_{1} \neq r_{2}$. Finally, suppose that $\left|\left(\zeta^{\prime} / \zeta\right)\left(s_{r}\right)\right| \geqslant \eta \log T$ for $1 \leqslant r \leqslant R$. Then

$$
R \ll T(\log T)^{3} \exp \left(-(f(\eta)-\varepsilon)\left(\alpha-\frac{1}{2}\right)(\log T) \log \left(\left(\alpha-\frac{1}{2}\right)(\log T)\right)\right),
$$

where $f(\eta)=1 /(\psi+\log (1+1 / 2 \eta))$. Here $\psi$ is the unique real number such that $e^{-\psi}+1=\psi$.

By Newton's method it is easily found that $\psi=1.27846 \ldots$

Proof. Since $\psi>1$, it is clear that $f(\eta)<1$ for any choice of $\eta$. Thus the bound to be proved is worse than the trivial bound $R \ll T$ if

$$
\frac{1}{2}+\frac{1}{\log T} \leqslant \alpha \leqslant \frac{1}{2}+\frac{\log \log T}{(\log T) \log \log \log T} .
$$

Hence we may assume that

$$
\alpha \geqslant \frac{1}{2}+\frac{\log \log T}{(\log T) \log \log \log T}
$$

Let

$$
w(n)= \begin{cases}1 & \text { if } n \leqslant u \\ \frac{\log u v / n}{\log v} & \text { if } u<n \leqslant u v \\ 0 & \text { if } n>u v\end{cases}
$$

Then

$$
\sum_{n} \frac{\Lambda(n) w(n)}{n^{s}}=\frac{-1}{2 \pi i} \int_{c-i \infty}^{c+i \infty} \frac{\zeta^{\prime}}{\zeta}(s+z) \frac{u^{z}\left(v^{z}-1\right)}{z^{2} \log v} d z .
$$

We move the contour to the left and apply the calculus of residues to see that the above is equal to

$$
-\frac{\zeta^{\prime}}{\zeta}(s)+\sum_{\rho} \frac{u^{\rho-s}\left(1-v^{\rho-s}\right)}{(\rho-s)^{2} \log v}+\frac{u^{1-s}\left(v^{1-s}-1\right)}{(s-1)^{2} \log v}+\sum_{k=1}^{\infty} \frac{u^{-2 k-s}\left(1-v^{-2 k-s}\right)}{(2 k+s)^{2} \log v},
$$

provided that $s \neq 1$, and that $\zeta(s) \neq 0$. In the case $u=v$, this is Lemma 2 of Selberg [12]. For a more elaborate formula of this kind, see [14, Lemma 10]. The above is true unconditionally, but if we assume $\mathrm{RH}$, then we find that the second term above has modulus at most

$$
\frac{u^{1 / 2-\sigma}\left(1+v^{1 / 2-\sigma}\right)}{(\sigma-1 / 2) \log v} \sum_{\rho} \frac{\sigma-1 / 2}{(\sigma-1 / 2)^{2}+(t-\gamma)^{2}} .
$$


Here the sum is $\Re\left(\xi^{\prime} / \xi\right)(s)$, which by Lemma 2 is at most $\Re\left(\zeta^{\prime} / \zeta\right)(s)+(1 / 2) \log (t / 2)$. Thus the above is at most

$$
\frac{u^{1 / 2-\alpha}\left(1+v^{1 / 2-\alpha}\right)}{(\alpha-1 / 2) \log v}\left(\Re \frac{\zeta^{\prime}}{\zeta}(s)+\frac{1}{2} \log T\right)
$$

for $\sigma \geqslant \alpha$ and $T \leqslant t \leqslant 2 T$.

Write $S(s)=\sum_{p \leqslant u v} w(p)(\log p) p^{-s}$. We must choose $u$ and $v$ to be sufficiently large so as to ensure that $\left|S\left(s_{r}\right)\right| \geqslant \delta \log T$. We may assume that $u \geqslant 2, v \geqslant 2$, and that $u v \leqslant T$. Thus the last two terms in (17) are much less than $1 / T$. Also,

$$
\sum_{p} \sum_{k=2}^{\infty} \frac{w\left(p^{k}\right) \log p}{p^{k s}} \ll \sum_{p} \frac{\log p}{p^{2 \alpha}} \ll \frac{1}{2 \alpha-1} \ll \frac{(\log T) \log \log \log T}{\log \log T}=\mathrm{o}(\log T)
$$

by (15), and so the quantity on the left has absolute value at most $\delta \log T$ for all sufficiently large $T$. Hence, if $\left|\left(\zeta^{\prime} / \zeta\right)\left(s_{r}\right)\right| \geqslant \eta \log T$, then $\left|S\left(s_{r}\right)\right| \geqslant \delta \log T$, provided that

$$
\eta\left(1-\frac{u^{1 / 2-\alpha}\left(1+v^{1 / 2-\alpha}\right)}{(\alpha-1 / 2) \log v}\right)-\frac{u^{1 / 2-\alpha}\left(1+v^{1 / 2-\alpha}\right)}{(2 \alpha-1) \log v} \geqslant 2 \delta .
$$

We want to take $k$ in Lemma 5 as large as possible. Therefore we want the above to hold with $u v$ as small as possible. In order to determine the optimal choice of these parameters, we find it convenient to introduce a change of variables as follows:

$$
u=\exp \left(\frac{U}{\alpha-1 / 2}\right), \quad v=\exp \left(\frac{V}{\alpha-1 / 2}\right) .
$$

Then (19) is equivalent to the inequality

$$
\frac{\eta-2 \delta}{\eta+1 / 2} \geqslant \frac{e^{-U}\left(1+e^{-V}\right)}{V}
$$

and we want $U+V$ to be as small as possible. We take $U$ such that the above holds with equality, since it would be wasteful to take $U$ any larger than necessary. Then

$$
U+V=\log \left(\frac{1+e^{-V}}{V}\right)+\log \left(\frac{\eta+1 / 2}{\eta-2 \delta}\right)+V .
$$

This is minimized by taking $V=\psi$, where $\psi$ is the unique real number such that $1+e^{-\psi}=\psi$. These considerations lead us to the choice

$$
u=\left(\frac{\eta+1 / 2}{\eta-2 \delta}\right)^{1 /(\alpha-1 / 2)}, \quad v=\exp \left(\frac{\psi}{\alpha-1 / 2}\right) .
$$

We take $k=[(\log T) / \log u v]$ in Lemma 5 . If $\delta$ is sufficiently small as a function of $\varepsilon$, then

$$
k \geqslant\left(f(\eta)-\frac{\varepsilon}{2}\right)\left(\alpha-\frac{1}{2}\right)(\log T) .
$$

Now

$$
\sum_{p} \frac{(\log p)^{2}}{p^{2 \alpha}} \ll \frac{1}{(2 \alpha-1)^{2}} .
$$

Indeed, with a little work it should be possible to show that the left-hand side above is strictly less than the right-hand side (that is, that the implicit constant can be taken to be 1), for all $\alpha>1 / 2$. Since $k ! \leqslant k^{k}$, by Lemma 5 it follows that

$$
(\delta \log T)^{2 k} R \ll T(\log T)^{2}\left(\frac{C k}{(2 \alpha-1)^{2}}\right)^{k} .
$$


Thus

$$
R \ll T(\log T)^{2}\left(\frac{C k}{\delta^{2}(2 \alpha-1)^{2}(\log T)^{2}}\right)^{k} \ll T(\log T)^{2}\left(\frac{C}{\delta^{2}(2 \alpha-1) \log T}\right)^{k} .
$$

This gives the stated result, in view of (15) and (20).

Lemma 7 (Assume RH). For $1 \leqslant r \leqslant R$ let $s_{r}=\sigma_{r}+i t_{r}$ be points such that $\sigma_{r} \geqslant \alpha \geqslant$ $1 / 2+10(\log \log T) / \log T, T \leqslant t_{r} \leqslant 2 T,\left|t_{r_{1}}-t_{r_{2}}\right| \geqslant 1$ when $r_{1} \neq r_{2}$, and

$$
\left|\log \zeta\left(s_{r}\right)\right| \geqslant\left(\alpha-\frac{1}{2}\right) \log T, \quad \Re \frac{\zeta^{\prime}}{\zeta}\left(s_{r}\right) \leqslant \frac{1}{2} \log T .
$$

Then

$$
R \ll T(\log T)^{3} \exp \left(-\frac{1}{2}\left(\alpha-\frac{1}{2}\right)(\log T) \log \frac{(\alpha-1 / 2) \log T}{2 \log \log T}\right)
$$

Proof. In (17) we replace $s$ by $s+x$, where $0 \leqslant x \leqslant 1$. The second term on the right-hand side has absolute value not exceeding

$$
\begin{gathered}
\frac{u^{1 / 2-\sigma-x}\left(1+v^{1 / 2-\sigma-x}\right)}{\log v} \sum_{\rho} \frac{1}{(\sigma+x-1 / 2)^{2}+(t-\gamma)^{2}} \\
\leqslant \frac{u^{1 / 2-\sigma-x}\left(1+v^{1 / 2-\sigma-x}\right)}{(\sigma-1 / 2) \log v} \sum_{\rho} \frac{\sigma-1 / 2}{(\sigma-1 / 2)^{2}+(t-\gamma)^{2}} .
\end{gathered}
$$

Here the last sum is $\Re\left(\xi^{\prime} / \xi\right)(s)$. Hence, by Lemma 2, the above is at most

$$
\frac{u^{1 / 2-\sigma-x}+(u v)^{1 / 2-\sigma-x}}{(\sigma-1 / 2) \log v}\left(\Re \frac{\zeta^{\prime}}{\zeta}(s)+\frac{1}{2} \log T\right)
$$

for $T \leqslant t \leqslant 2 T$. We integrate over $0 \leqslant x \leqslant 1$ to see that

$$
\left|\log \zeta(s)-\sum_{n} \frac{\Lambda(n) w(n)}{(\log n) n^{s}}\right| \leqslant\left(\frac{u^{1 / 2-\alpha}}{\log u}+\frac{(u v)^{1 / 2-\alpha}}{\log u v}\right) \frac{\Re\left(\zeta^{\prime} / \zeta\right)(s)+(1 / 2) \log T}{(\alpha-1 / 2) \log v}+\mathrm{O}(1) .
$$

We now take $u=v=\exp (1 /(\alpha-1 / 2))$. Thus, if $\Re\left(\zeta^{\prime} / \zeta\right)(s) \leqslant(1 / 2) \log T$, then the above is less than $(9 / 20)(\alpha-1 / 2) \log T$. Write $S(s)=\sum_{p} w(p) p^{-s}$. We note that

$$
\begin{aligned}
\left|\sum_{p} \sum_{k=2}^{\infty} \frac{w\left(p^{k}\right)}{k p^{k s}}\right| & \leqslant \frac{1}{2} \sum_{p \leqslant u v} \frac{1}{p}+\mathrm{O}(1) \leqslant \frac{1}{2} \log \frac{2}{\alpha-1 / 2}+\mathrm{O}(1) \\
& \leqslant \frac{1}{2} \log \log T \leqslant \frac{1}{20}\left(\alpha-\frac{1}{2}\right) \log T
\end{aligned}
$$

Thus we see that if $(21)$ holds then $\left|S\left(s_{r}\right)\right| \geqslant(1 / 2)(\alpha-1 / 2) \log T$. We apply Lemma 5 with $k=[(1 / 2)(\alpha-1 / 2) \log T]$. Thus we find that

$$
\left(\frac{1}{2}\left(\alpha-\frac{1}{2}\right) \log T\right)^{2 k} R \ll T(\log T)^{2} k !\left(\sum_{p} \frac{w(p)^{2}}{p^{2 \alpha}}\right)^{k} .
$$

Now

$$
\sum_{p} \frac{w(p)^{2}}{p^{2 \alpha}} \leqslant \sum_{p \leqslant u v} \frac{1}{p}=\log \frac{2}{\alpha-1 / 2}+\mathrm{O}(1) \leqslant \log \log T
$$


Hence

$$
\begin{aligned}
R & \ll T(\log T)^{2}\left(\frac{4 k \log \log T}{(\alpha-1 / 2)^{2}(\log T)^{2}}\right)^{k} \leqslant T(\log T)^{2}\left(\frac{2 \log \log T}{(\alpha-1 / 2) \log T}\right)^{k} \\
& \ll T(\log T)^{3}\left(\frac{2 \log \log T}{(\alpha-1 / 2) \log T}\right)^{(1 / 2)(\alpha-1 / 2) \log T} .
\end{aligned}
$$

This gives the stated result.

Lemma 8 (Assume RH). For $1 \leqslant r \leqslant R$ let $s_{r}=\sigma_{r}+i t_{r}$ be points such that $\sigma_{r} \geqslant 1 / 2, T \leqslant$ $t_{r} \leqslant 2 T$, and $\left|t_{r_{1}}-t_{r_{2}}\right| \geqslant 1$ when $r_{1} \neq r_{2}$. Suppose also that $V \geqslant 15 \log \log T$, that $\log \left|\zeta\left(s_{r}\right)\right| \leqslant$ $-V$, and that

$$
\Re \frac{\zeta^{\prime}}{\zeta}\left(\sigma+i t_{r}\right) \leqslant \frac{1}{2} \log T
$$

for $\sigma_{r} \leqslant \sigma<\infty$. Then

$$
R \ll T(\log T)^{3} \exp \left(-\frac{V}{3} \log \frac{V}{3 \log \log T}\right) .
$$

Proof. Write $\sigma_{r}^{\prime}=\sigma_{r}+2 V /(3 \log T)$, and set $s_{r}^{\prime}=\sigma_{r}^{\prime}+i t_{r}$. Then

$$
V+\log \left|\zeta\left(s_{r}^{\prime}\right)\right| \leqslant \log \left|\zeta\left(s_{r}^{\prime}\right)\right|-\log \left|\zeta\left(s_{r}\right)\right|=\int_{\sigma_{r}}^{\sigma_{r}^{\prime}} \Re \frac{\zeta^{\prime}}{\zeta}\left(\sigma+i t_{r}\right) d \sigma .
$$

By the bound (22), this is at most

$$
\left(\sigma_{r}^{\prime}-\sigma_{r}\right) \frac{1}{2} \log T=\frac{V}{3} .
$$

Hence $\log \left|\zeta\left(s_{r}^{\prime}\right)\right| \leqslant-2 V / 3$. Write $\alpha=1 / 2+2 V /(3 \log T)$, and note that $\sigma_{r}^{\prime} \geqslant \alpha$ for all $r$. The stated bound now follows from Lemma 7 with the $s_{r}$ replaced by the $s_{r}^{\prime}$.

\section{Proof of Theorem 1}

First we define the contour. The contour is to be symmetric with respect to the real axis, and so it suffices to describe it in the upper half-plane. Let $c=39 / 61$, and set $J=\left[(\log x)^{c} / \log 2\right]$. Thus $2^{J} \leqslant \exp \left((\log x)^{c}\right) \leqslant 2^{J+1}$. For $0 \leqslant t \leqslant 2^{J}$ we proceed along a polygonal path with vertices

$$
\begin{aligned}
& \frac{1}{2}+\frac{2}{\log x}, \quad \frac{1}{2}+\frac{2}{\log x}+16 i, \quad \frac{1}{2}+\frac{4}{(\log 4) \log x}+16 i, \quad \frac{1}{2}+\frac{4}{(\log 4) \log x}+32 i, \\
& \frac{1}{2}+\frac{5}{(\log 5) \log x}+32 i,
\end{aligned}
$$

and, in general,

$$
\ldots, \frac{1}{2}+\frac{j}{(\log j) \log x}+2^{j} i, \frac{1}{2}+\frac{j}{(\log j) \log x}+2^{j+1} i, \frac{1}{2}+\frac{j+1}{(\log (j+1)) \log x}+2^{j+1} i, \ldots
$$

until we reach the point

$$
\frac{1}{2}+\frac{J}{(\log J) \log x}+2^{J} i .
$$

For $j \geqslant J$ set $T=2^{j}$. We now define the contour for $T \leqslant t \leqslant 2 T$. For each integer $r$, where $T \leqslant r<2 T$, define $\sigma_{2}(r)$ to be the least number such that

$$
\Re \frac{\zeta^{\prime}}{\zeta}(s) \leqslant \eta \log T
$$


for all $s$ in the half-strip $\sigma \geqslant \sigma_{2}(r)$ and $r \leqslant t \leqslant r+1$. Here $\eta$ is a positive parameter whose value will be chosen later. For the present, we assume only that $0<\eta<1 / 2$. Let $1 / 2+i \gamma$ be a zero of the zeta function with $r \leqslant \gamma \leqslant r+1$. From (10) we see that $\Re\left(\xi^{\prime} / \xi\right)(s)>\log T$ when $s=1 / 2+1 / \log T+i \gamma$. From Lemma 2 it then follows that $\Re\left(\zeta^{\prime} / \zeta\right)(s)>(1 / 2) \log T>\eta \log T$ for this same $s$. Thus

$$
\sigma_{2}(r) \geqslant \frac{1}{2}+\frac{1}{\log T}
$$

for all $r$. With $\sigma_{2}(r)$ determined in this way, we write

$$
\sigma_{1}(r)=\frac{1}{2}+\left(\sigma_{2}(r)-\frac{1}{2}\right) \frac{\log T}{\log x} .
$$

To extend our contour from the point (23), we first move to the point $\sigma_{1}\left(2^{J}\right)+2^{J} i$. After that, for each $r \geqslant 2^{J}$, we move from $\sigma_{1}(r)+r i$ to $\sigma_{1}(r)+(r+1) i$, and from there to $\sigma_{1}(r+1)+$ $(r+1) i$.

We now prove $(2)$. Let $\gamma_{1}=14.13 \ldots$ and $\gamma_{2}=21.02 \ldots$ denote the ordinates of the first two zeros of the zeta function. Since $\zeta^{\prime}\left(1 / 2+i \gamma_{1}\right) \neq 0$, it follows that $|\zeta(s)| \asymp\left|s-1 / 2-i \gamma_{1}\right|$ for $s$ near $1 / 2+i \gamma_{1}$. Since $\gamma_{2}>16,(2)$ is immediate.

Next we prove (3). Suppose that $T=2^{j}$ with $4 \leqslant j \leqslant J$. From Littlewood's estimates (13) and Lemma 4 it is clear that

$$
\frac{1}{\zeta(s)} \ll\left(\frac{e \log x}{\log T}\right)^{\frac{C \log T}{\log \log T}}
$$

for $s \in$ with $T \leqslant t \leqslant 2 T$. Thus we have (3).

With these preliminaries completed, we initiate the proof of the main estimate, (4). Suppose that $T=2^{j}$ with $j \geqslant J$. For $T \leqslant r<2 T$, let $t_{1}(r)$ be chosen such that $\left|\zeta\left(\sigma_{1}(r)+i t\right)\right|$ takes its minimum, for $r \leqslant t \leqslant r+1$, at $t=t_{1}(r)$. Set $s_{1}(r)=\sigma_{1}(r)+i t_{1}(r)$, and set $m(r)=$ $1 /\left|\zeta\left(s_{1}(r)\right)\right|$. Then

$$
\int_{r<t<r+1}\left|\frac{x^{s}}{\zeta(s)}\right||d s|=\int_{r}^{r+1} \frac{x^{\sigma_{1}(r)}}{\left|\zeta\left(\sigma_{1}+i t\right)\right|} d t \leqslant x^{\sigma_{1}(r)} m(r) .
$$

Next we establish the last clause of Theorem 1. The logarithmic derivative of the expression in question is $\log x-\Re\left(\zeta^{\prime} / \zeta\right)(\sigma+i t)$. Suppose that $r \leqslant t \leqslant r+1$. By the definition of $\sigma_{2}(r)$, we know that

$$
\Re \frac{\zeta^{\prime}}{\zeta}(\sigma+i t) \leqslant \eta \log T \leqslant \frac{1}{2} \log T \leqslant \log x
$$

for $\sigma \geqslant \sigma_{2}(r)$ and $r \leqslant t \leqslant r+1$. As for the remaining range, $\sigma_{1}(r) \leqslant \sigma \leqslant \sigma_{2}(r)$, we note that, by Lemma 3 we have

$$
\begin{aligned}
\Re \frac{\zeta^{\prime}}{\zeta}(\sigma+i t) & \leqslant \Re \frac{\zeta^{\prime}}{\zeta}(\sigma+i t)+\frac{1}{2} \log \frac{t}{2} \leqslant \frac{\sigma_{2}(r)-1 / 2}{\sigma-1 / 2}\left(\Re \frac{\zeta^{\prime}}{\zeta}\left(\sigma_{2}(r)+i t\right)+\frac{1}{2} \log \frac{t}{2}\right) \\
& \leqslant \frac{\sigma_{2}(r)-1 / 2}{\sigma-1 / 2} \log T \leqslant \frac{\sigma_{2}(r)-1 / 2}{\sigma_{1}(r)-1 / 2} \log T=\log x .
\end{aligned}
$$

Since $x^{\sigma} /|\zeta(\sigma+i r)|$ is monotonically increasing for $\sigma \geqslant \min \left(\sigma_{1}(r-1), \sigma_{1}(r)\right)$, and since the interval from $\sigma_{1}(r-1)+i r$ to $\sigma_{1}(r)+i r$ has length at most 1 , it follows that

$$
\int_{t=r}\left|\frac{x^{s}}{\zeta(s)}\right||d s| \leqslant m(r-1) x^{\sigma_{1}(r-1)}+m(r) x^{\sigma_{1}(r)} .
$$


On combining this with (26), we deduce that

$$
\int_{T<t \leqslant 2 T}\left|\frac{x^{s}}{\zeta(s)}\right||d s| \ll \sum_{r=T}^{2 T} x^{\sigma_{1}(r)} m(r) .
$$

From the definition of $\sigma_{1}(r)$, it is clear that $x^{\sigma_{1}}=x^{1 / 2} T^{\sigma_{2}(r)-1 / 2}$. Also, by Lemma 4 we see that

$$
m(r)=\frac{1}{\left|\zeta\left(\sigma_{1}(r)+i t_{1}(r)\right)\right|} \leqslant \frac{(\log x / \log T)^{\left(\sigma_{2}(r)-1 / 2\right)(\eta+1 / 2) \log T}}{\left|\zeta\left(\sigma_{2}(r)+i t_{1}(r)\right)\right|}
$$

since $\Re\left(\zeta^{\prime} / \zeta\right)\left(\sigma_{2}(r)+i t_{1}(r)\right) \leqslant \eta \log T$. On combining this with $(27)$, we deduce that

$$
\int_{T<t \leqslant 2 T}\left|\frac{x^{s}}{\zeta(s)}\right||d s| \ll x^{1 / 2} \sum_{r=T}^{2 T} \frac{(8 \log x / \log T)^{\left(\sigma_{2}(r)-1 / 2\right)(\eta+1 / 2) \log T}}{\left|\zeta\left(\sigma_{2}(r)+i t_{1}(r)\right)\right|} .
$$

To estimate the right-hand side above, we consider three types of $r$. Let ${ }_{1}$ denote the set of those $r$, where $T \leqslant r \leqslant 2 T$, for which

$$
\frac{1}{\left|\zeta\left(\sigma_{2}(r)+i t_{1}(r)\right)\right|} \leqslant(\log T)^{15} \exp \left(\varepsilon\left(\sigma_{2}(r)-\frac{1}{2}\right)(\log T) \log \left(\left(\sigma_{2}(r)-\frac{1}{2}\right) \log T\right)\right) .
$$

Let 2 denote the set of those $r$, where $T \leqslant r \leqslant 2 T$, for which

$$
\sigma_{2}(r) \geqslant \frac{1}{2}+\frac{C_{1} \log \log T}{(\log T) \log \log \log T}
$$

and

$$
\frac{1}{\left|\zeta\left(\sigma_{2}(r)+i t_{1}(r)\right)\right|}>\exp \left(\varepsilon\left(\sigma_{2}(r)-\frac{1}{2}\right)(\log T) \log \left(\left(\sigma_{2}(r)-\frac{1}{2}\right) \log T\right)\right) .
$$

Here $C_{1}=C_{1}(\varepsilon)$ is a large constant whose value will be determined later. Finally, let ${ }_{3}$ denote the set of those $r$, where $T \leqslant r \leqslant 2 T$, for which

$$
\sigma_{2}(r) \leqslant \frac{1}{2}+\frac{C_{1} \log \log T}{(\log T) \log \log \log T}
$$

and

$$
\frac{1}{\left|\zeta\left(\sigma_{2}(r)+i t_{1}(r)\right)\right|}>(\log T)^{15} .
$$

We note that, if (30) holds but (31) fails, then $r \in 1$, and that, if (32) holds but (33) fails, then $r \in{ }_{1}$. Thus every $r$ is in at least one of the $i$.

For $r \in 1_{1}$, choose $t_{2}(r)$, where $r \leqslant t_{2}(r) \leqslant r+1$, such that $\Re\left(\zeta^{\prime} / \zeta\right)\left(\sigma_{2}(r)+i t_{2}(r)\right)=$ $\eta \log T$. Among the $r \in{ }_{1}$, consider those for which $\alpha \leqslant \sigma_{2}(r)<\alpha+\delta$, where $\delta=(\log T)^{-2}$. By Lemma 6, the contribution of these $r$ to (28) is at most of the order of

$$
x^{1 / 2} T(\log T)^{18} \exp (g(\alpha)),
$$

where

$g(\alpha)=\left(\alpha-\frac{1}{2}\right)(\log T)\left(\eta+\frac{1}{2}\right) \log \frac{8 \log x}{\log T}-(f(\eta)-2 \varepsilon)\left(\alpha-\frac{1}{2}\right)(\log T) \log \left(\left(\alpha-\frac{1}{2}\right) \log T\right)$.

This function assumes its maximum at

$$
\alpha=\frac{1}{2}+\frac{1}{e \log T}\left(\frac{8 \log x}{\log T}\right)^{(\eta+1 / 2) /(f(\eta)-2 \varepsilon)} .
$$

The maximum value attained is

$$
\frac{f(\eta)-2 \varepsilon}{e}\left(\frac{8 \log x}{\log T}\right)^{(\eta+1 / 2) /(f(\eta)-2 \varepsilon)} .
$$


This motivates us to take $\eta$ so as to minimize the above exponent; that is, we take $\eta$ to be the unique real number such that

$$
\psi+\log \left(1+\frac{1}{2 \eta}\right)=\frac{1}{2 \eta} .
$$

Numerically, $\eta=0.196570958763 \ldots, f(\eta)=0.393141917526 \ldots$, and $(\eta+1 / 2) / f(\eta)=1 / 2+$ $1 /(4 \eta)=1.771805365213 \ldots<39 / 22$. We also set $\varepsilon=10^{-4}$, and observe that $(\eta+$ $1 / 2) /(f(\eta)-2 \varepsilon)<39 / 22$. On summing over $\alpha=1 / 2+1 / \log T+k \delta$, we conclude that the total contribution of all $r \in 1$ is at most of the order of

$$
x^{1 / 2} T(\log T)^{20} \exp \left(\left(\frac{\log x}{\log T}\right)^{39 / 22}\right) .
$$

Among the $r \in{ }_{2}$, we consider those for which $\alpha \leqslant \sigma_{2}(r)<\alpha+\delta$ and $V \leqslant-\log \mid \zeta\left(\sigma_{2}(r)+\right.$ $\left.i t_{1}(r)\right) \mid<2 V$, where

$$
\alpha \geqslant \frac{1}{2}+\frac{C_{1} \log \log T}{(\log T) \log \log \log T}, \quad V \geqslant V_{0}=\varepsilon\left(\alpha-\frac{1}{2}\right)(\log T) \log \left(\left(\alpha-\frac{1}{2}\right) \log T\right) .
$$

We now take $C_{1}=(4 / \varepsilon) \exp (9 / \varepsilon)$. This ensures that

$$
\log \frac{V}{3 \log \log T} \geqslant \frac{9}{\varepsilon} \text {. }
$$

Hence, by Lemma 8, the number of such $r$ is at most of the order of $T(\log T)^{3} \exp (-3 V / \varepsilon)$. Since $1 /\left|\zeta\left(\sigma_{2}(r)+i t_{1}(r)\right)\right| \leqslant \exp (2 V)$ for these $r$, on summing over $V=V_{0} 2^{k}$ we deduce that the total contribution to (28) of those $r \in{ }_{2}$ for which $\alpha \leqslant \sigma_{2}(r)<\alpha+\delta$ is at most of the order of $x^{1 / 2} T(\log T)^{3} \exp (h(\alpha))$, where

$$
h(\alpha)=\left(\alpha-\frac{1}{2}\right)(\log T)\left(\eta+\frac{1}{2}\right) \log \frac{8 \log x}{\log T}-\left(\alpha-\frac{1}{2}\right)(\log T) \log \left(\left(\alpha-\frac{1}{2}\right) \log T\right) .
$$

This is of the same form as the function $g(\alpha)$ that arose in the preceding case, but with a more favorable constant. By proceeding as in the former case, we find that the total contribution to (28) of all $r \in{ }_{2}$ is at most of the order of

$$
x^{1 / 2} T(\log T)^{5} \exp \left(\frac{\log x}{\log T}\right) .
$$

Suppose that $V \geqslant 15 \log \log T$, and consider those $r \in{ }_{3}$ for which $V \leqslant-\log \mid \zeta\left(\sigma_{2}(r)+\right.$ $\left.i t_{1}(r)\right) \mid<(51 / 50) V$. By Lemma 8, the sum of $1 /\left|\zeta\left(\sigma_{2}(r)+i t_{1}(r)\right)\right|$ over such $r$ is at most of the order of

$$
T(\log T)^{3} \exp \left(\frac{51}{50} V-\frac{V}{3 \log \log T}\right) \text {. }
$$

On summing this over $V=15(51 / 50)^{k} \log \log T$, we find that

$$
\sum_{r \in \in_{3}} \frac{1}{\left|\zeta\left(\sigma_{2}(r)+i t_{1}(r)\right)\right|} \ll T(\log T)^{11} .
$$

On the other hand, for $r \in 3$ we have

$$
\left(\frac{8 \log x}{\log T}\right)^{\left(\sigma_{2}(r)-1 / 2\right)(\eta+1 / 2) \log T} \leqslant\left(\frac{8 \log x}{\log T}\right)^{C_{1}(\log \log T) / \log \log \log T} .
$$

The ratio of the above with $\exp \left(((\log x) / \log T)^{39 / 22}\right)$ is largest when $(\log x) / \log T$ is of the form

$$
C\left(\frac{\log \log T}{\log \log \log T}\right)^{22 / 39}
$$


and therefore the right-hand side of (37) is at most of the order of

$$
(\log T)^{A} \exp \left(\left(\frac{\log x}{\log T}\right)^{39 / 22}\right) .
$$

Hence the total contribution of all $r \in{ }_{3}$ to (28) is at most of the order of

$$
x^{1 / 2} T(\log T)^{A} \exp \left(\left(\frac{\log x}{\log T}\right)^{39 / 22}\right) .
$$

On combining this with (35) and (36) in (28), we obtain (4), and the proof is complete.

\section{Proof of the corollaries}

By the truncated form of Perron's formula (see [9, Corollary 5.3]), we know that

$$
M(x)=\frac{1}{2 \pi i} \int_{c-i T}^{c+i T} \frac{x^{s}}{\zeta(s) s} d s+\mathrm{O}\left(\frac{x \log x}{T}\right),
$$

where $c=1+1 / \log x$. Let $\sigma(T)$ be chosen such that $\sigma(T)+i T \in$. By the last clause of the Theorem 1, we know that

$$
\int_{\sigma(T)+i T}^{c+i T} \frac{x^{s}}{\zeta(s) s} d s \ll \frac{x \log x}{T}
$$

Hence

$$
M(x)=\frac{1}{2 \pi i} \int_{-T \leqslant t \leqslant T} \frac{x^{s}}{\zeta(s) s} d s+\mathrm{O}\left(\frac{x \log x}{T}\right) .
$$

We take $T=x^{1 / 2}$, and apply the estimates (2)-(4) to obtain Corollary 1.

In proving Corollary 2 , we may assume that $h \leqslant x^{3 / 4}$, as otherwise the stated bound follows directly from Corollary 1. From (38) we see that

$$
M(x+h)-M(x)=\frac{1}{2 \pi i} \int_{-T \leqslant t \leqslant T} \frac{(x+h)^{s}-x^{s}}{\zeta(s) s} d s+\mathrm{O}\left(\frac{x \log x}{T}\right) .
$$

Clearly,

$$
\frac{(x+h)^{s}-x^{s}}{s} \ll \begin{cases}h x^{\sigma-1} & (|t| \leqslant x / h), \\ x^{\sigma} /|t| & (|t| \geqslant x / h) .\end{cases}
$$

Hence from (2)-(4) we see that

$$
M(x+h)-M(x) \ll \delta x^{1 / 2}(\log x)^{A} .
$$

This suffices.

Acknowledgement. The authors are grateful to Adolf Hildebrand for his helpful remarks.

\section{References}

1. C. P. Hughes, J. P. Keating, and N. O'Connell, 'Random matrix theory and the derivative of the Riemann zeta function', Proc. R. Soc. London Ser. A Math. Phys. Eng. Sci. 456 (2000) 2611-2627.

2. E. Landau, 'Über die Möbiussche Funktion', Rend. Circ. Mat. Palermo 48 (1924) 277-280.

3. J. E. LitTlewood, 'Quelques conséquences de l'hypothèse que la fonction $\zeta(s)$ de Riemann n'a pas de zéros dans le demi-plan $\Re(s)>1 / 2$ ', C. R. Math. Acad. Sci. Paris 154 (1912) 263-266.

4. J. E. Littlewood, 'On the zeros of the Riemann zeta-function', Proc. Cambridge Philos. Soc. 22 (1924) $295-318$. 
5. H. L. Montgomery, Topics in multiplicative number theory, Lecture Notes in Mathematics 227 (Springer, Berlin, 1971).

6. H. L. Montgomery, 'The analytic principle of the large sieve', Bull. Amer. Math. Soc. 84 (1978) $547-567$.

7. H. L. Montgomery, 'The zeta function and prime numbers', Proceedings of the Queen's Number Theory Conference, Kingston, Ontario, 1979, Queen's Papers in Pure and Applied Mathematics 54 (ed. P. Ribenboim; Queen's University, Kingston, 1980), 1-31.

8. H. L. Montgomery and R. C. Vaughan, 'Hilbert's inequality', J. London Math. Soc. (2) 8 (1974) 73-82.

9. H. L. Montgomery and R. C. Vaughan, Multiplicative number theory I. Classical theory, Cambridge Studies in Advanced Mathematics 97 (Cambridge University Press, Cambridge, 2007).

10. N. NG, 'The distribution of the summatory function of the Möbius function', Proc. London Math. Soc. (3) 89 (2004) 361-389.

11. A. M. OdLyzko and H. J. J. te Riele, 'Disproof of the Mertens conjecture', J. Reine Angew. Math. 357 (1985) 138-160.

12. A. SElberg, 'On the normal density of primes in small intervals', Archiv for Mathematik og Naturvidenskab 47, Collected Papers 1 (Springer, Berlin, 1989) 160-178.

13. A. SelBerg, 'On the remainder in the formula for $N(T)$, the number of zeros of $\zeta(s)$ in the strip $0<t<T$ ', Avhandl. Norske Vid. Akad. Oslo I. Mat.-Naturv. Klasse (1944), No. 1, 1-27.

14. A. Selberg, 'Contributions to the theory of the Riemann zeta-function', Archiv for Mathematik og Naturvidenskab 48, Collected Papers 1 (Springer, Berlin, 1989) 214-280.

15. E. C. Titchmarsh, 'A consequence of the Riemann hypothesis', J. London Math. Soc. 2 (1927) $247-254$.

\section{H. Maier}

Abteilung Zahlentheorie und

Wahrscheinlichkeitstheorie

Helmholtzstrasse 18

Universität Ulm

89069 Ulm

Germany

helmut.maier@uni-ulm.de
H. L. Montgomery

Department of Mathematics

University of Michigan

Ann Arbor, MI 48109-1043

USA

hlm@umich.edu 